\title{
ARTICLE
}

\section{Cavitary retinoblastoma: clinical observations}

\author{
Pukhraj Rishi $\mathbb{1}^{1} \cdot$ Unnati Sharma $\mathbb{1}^{1} \cdot$ Tarun Sharma ${ }^{1}$
}

Received: 16 April 2019 / Revised: 29 July 2019 / Accepted: 13 August 2019 / Published online: 18 September 2019

(c) The Author(s), under exclusive licence to The Royal College of Ophthalmologists 2019

\begin{abstract}
Aim A retrospective case series describing the clinical features and treatment outcomes in eye with cavitary retinoblastoma. Methods Case records of patients diagnosed with cavitary retinoblastoma from 2013 to 2017 were reviewed and their demographic details, clinical presentation, and treatment outcomes were analysed.

Results Thirteen tumours from ten eyes of ten patients were included. Mean age at diagnosis was 36 months (median $=30$, range $=2-60$ months). Mean number of cavities per tumour were two (median 1, range 1-5). Sixty-two percent of tumours had primary cavities, $23 \%$ had secondary cavities, while $15 \%$ had both types. Mean basal tumour diameter at presentation was $10.9 \mathrm{~mm}$, and at final follow-up was $10.4 \mathrm{~mm}$. Mean tumour thickness at presentation was $7.7 \mathrm{~mm}$, and at final followup was $6.5 \mathrm{~mm}$. Majority of tumours (46\%) showed type 2 regression pattern. Tumour recurrence was noted in $1(8 \%)$ eye. Cavity rupture with release of vitreous seeds was observed in one eye. Two (20\%) eyes with vitreous seeds were treated with intravitreal chemotherapy. Two eyes were advised enucleation, one due to tumour recurrence and the other due to persistent vitreous seeds. No patients had metastasis or death. Mean follow-up was 54 months (median 20, SD 66.82, range 3-183). Conclusion Cavitary tumours have variable presentations, are often associated with vitreous seeds, and in some cases the latter emanates from them as well. Cavitary tumours tend to maintain stable tumour dimensions.
\end{abstract}

\section{Introduction}

Retinoblastoma is a common intraocular malignancy of childhood, usually presenting before 5 years of age. The typical features on clinical examination comprise of yellowish-white solid retinal mass with or without vitreous seeds, subretinal seeds, and subretinal fluid. Cavitary retinoblastoma is a variant of retinoblastoma characterized by the presence of ophthalmoscopically visible translucent cavities within the tumour mass [1]. There is scarce published literature describing cavitary retinoblastoma. PubMed search using keyword (Cavitary retinoblastoma) revealed five relevant studies. Hereby, we present our study describing the clinical features, disease course, and management outcomes of cavitary retinoblastoma.

Pukhraj Rishi

docrishi@yahoo.co.in

1 Shri Bhagwan Mahavir Vitreoretinal Services, Sankara Nethralaya, 18 College Road, Chennai, Tamil Nadu 600006, India

\section{Method and materials}

A retrospective review of medical records for patients diagnosed with retinoblastoma from June 2013 to November 2017 was carried out and cases with 'tumour cavities' detected at presentation or during the course of follow-up were included. A written informed consent was signed by the parents of all patients for examination, treatment and use of the relevant data for research. This study adhered to the tenets of the Declaration of Helsinki and prior institutional review board approval was sought. Case records were screened for demographic details, ocular examination findings, tumour associated features, treatment, complications, and follow-up. Data collected on continuous and ordinal scale were expressed as mean, median, and range. Visual acuity was recorded as 'fixates and follows' or 'does not fixate and follow' in patients who could not read on Snellen's chart. Tumour dimensions were measured in millimetres using callipers on ultrasound scan (Alcon Laboratories, Fort Worth, TX, USA).

Tumours were classified according to international classification of retinoblastoma (ICRB) [2]. 'Cavitary retinoblastoma' was defined as retinoblastoma tumour consisting of translucent cystoid or cavitary spaces on clinical examination. 'Primary cavities' were the ones that presented 
in treatment naïve tumours, while 'secondary cavities' were defined as the ones that became clinically apparent during the course of tumour management. The term 'peripheral cavities' was used for those located within peripheral $1 / 3$ of tumour surface, while central cavities were defined as cavities located within central $2 / 3$ of tumour surface [1]. Differences in tumour dimensions at baseline and follow-up were statistically compared by applying paired $t$-test using SPSS version 14. $P$ value $<0.05$ was considered significant.

\section{Results}

Thirteen tumours in ten eyes of ten patients were included in the study between June 2013 and November 2017. Table 1 shows the clinical details of patients at presentation. Seven patients $(70 \%)$ presented with leucocoria, one $(10 \%)$ with strabismus, and two (20\%) with redness and swelling of eyes. Anterior segment examination was normal in majority $(n=8,80 \%)$ of eyes with cavitary retinoblastoma, while two (20\%) eyes had neovascularization of iris and one (10\%) eye had lens haze. None of the eyes had AC seeds. Visual acuity examinations showed normal 'fixates and follows' light in three (30\%) eyes, while four (40\%) did not 'fixate and follow' light. Three patients were in the age group 4-5 years, had visual acuity of hand movements $(n=$ $1,10 \%), 2 / 60(n=1,10 \%)$, and $6 / 36(n=1,10 \%)$. Majority of patients $(70 \%)$ had bilateral tumours. Six patients had fellow eye enucleated for advanced retinoblastoma. Mean number of tumours per eye was 2 (median $=2$, range 1-3), while mean number of cavitary tumour(s) per eye was 1 (median $=1$, range 1-2). Three eyes had two cavitary tumours, while others harboured single cavitary tumour, each. Majority $(n=10,77 \%)$ of tumours had single cavities, while three (23\%) tumours had multiple cavities. Mean number of cavities per tumour was 2 (median 1, range 1-5, SD 1.2). Of 20 cavities, 14 (70\%) cavities were located in tumour periphery, while six (30\%) were central in location. Sixteen ( $80 \%)$ of 20 cavities were 'primary', while the other four $(20 \%)$ were 'secondary' in nature. Secondary cavities became evident either after one cycle of intravenous chemotherapy $(n=1)$, after two cycles $(n=1)$, after four cycles of intravenous chemotherapy $(n=1)$ or even after EBRT $(n=1)$. Mean number of chemotherapy session after which the secondary cavities were clinically evident were 2 (median 2, SD 1.26). One tumour (Case 8) had both primary and secondary cavity; secondary cavity being noticeable after two cycles of intra-arterial chemotherapy.

Four eyes $(40 \%)$ had vitreous seed at the time of presentation. Of these, one eye had two tumours, one of which was cavitary, while the other was non-cavitary; one eye had single cavitary tumour that harboured primary and
Table 1 Baseline clinical features of 10 eyes with cavitary RB

\begin{tabular}{|c|c|}
\hline & $(\%)$ \\
\hline \multicolumn{2}{|l|}{ ICRB classification $(n=10)$} \\
\hline Group B & $3(30)$ \\
\hline Group C & $4(40)$ \\
\hline Group D & $3(30)$ \\
\hline \multicolumn{2}{|l|}{ Type of tumour $(n=13)$} \\
\hline Endophytic & $5(39)$ \\
\hline Exophytic & $6(46)$ \\
\hline Mixed & $2(15)$ \\
\hline \multicolumn{2}{|l|}{ Location of cavities $(n=13)$} \\
\hline Central & $2(15)$ \\
\hline Peripheral & $8(62)$ \\
\hline Central and peripheral & $3(23)$ \\
\hline \multicolumn{2}{|l|}{ Type of cavities $(n=13)$} \\
\hline Primary & $9(69)$ \\
\hline Secondary & $3(23)$ \\
\hline Primary and secondary & $1(8)$ \\
\hline \multicolumn{2}{|l|}{ Location of tumour $(n=13)$} \\
\hline Disc & $2(15)$ \\
\hline Macula (within arcade) & $3(23)$ \\
\hline Superior to disc & $1(8)$ \\
\hline STQ arcade & $2(15)$ \\
\hline Inferior to disc & $1(8)$ \\
\hline INQ to disc & $1(8)$ \\
\hline Nasal to disc & $3(23)$ \\
\hline \multicolumn{2}{|l|}{ Associated findings } \\
\hline Vitreous seeds $(n=10)$ & $4(40)$ \\
\hline Subretinal fluid $(n=10)$ & $4(40)$ \\
\hline Subretinal seeds $(n=10)$ & $1(10)$ \\
\hline Calcification $(n=13)$ & $3(30)$ \\
\hline
\end{tabular}

ICRB international classification of retinoblastoma, $S T Q$ superotemporal quadrant, $I N Q$ Inferonasal quadrant

secondary cavities; one eye had two tumours- of which one was primary cavitary and the other was secondary cavitary, while one eye had two primary cavitary tumours. One tumour showed vitreous seeds after cavity rupture (Case 1; Fig. 1) following two cycles of chemotherapy and was treated with intravitreal chemotherapy (one dose of Intravitreal melphalan-20 $\mu \mathrm{g} / 0.02 \mathrm{ml}$ ). At presentation, mean tumour calcification noted was $15 \%$, while at final followup, it was $20 \%$. Table 2 shows the individual details for the ten eyes in the study. Most tumours in our study were primarily treated with systemic chemotherapy with focal consolidation (Fig. 2). Two (20\%) eyes with vitreous seeds were treated with intravitreal chemotherapy (Fig. 3). Mean duration of follow-up was 54 months (median 20, range $3-183$, SD 66.82). None of the tumour showed type 1 or 4 regression pattern. Six of thirteen (46\%) tumours showed 


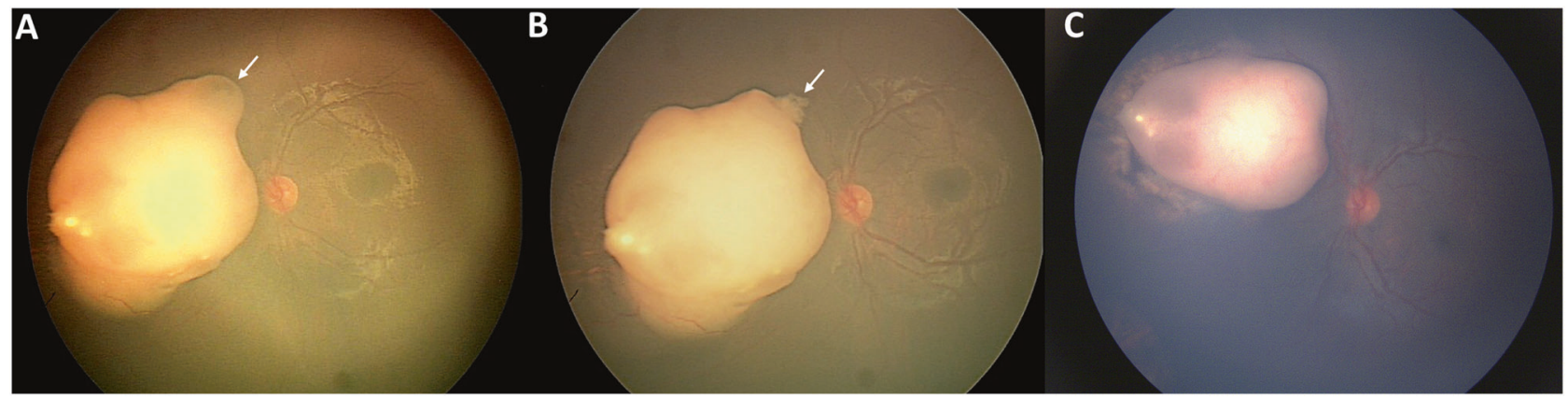

Fig. 1 At presentation (a), left eye fundus photo shows group $\mathrm{C}$ retinoblastoma with cavitary tumour having peripheral cavities (arrow). On a follow-up after three cycles of intravenous chemotherapy (b) and immediately following cryotherapy, release of vitreous seeds from the tumour 'cavity' is seen (arrow); intravitreal chemotherapy was given. Tumour regression after six cycles of chemotherapy (c) type 2 regression pattern, while four (31\%) tumours showed type 3 regression. Two tumours (15\%) were diagnosed as retinoma. In one eye where the tumour was excised during surgery for rhegmatogenous retinal detachment, did not show any recurrence or metastasis after 13 years of followup. Globe salvage was achieved in $80 \%(n=8)$ eyes.

All except two eyes could be salvaged in our study. Of these, one eye had two tumours of which one was cavitary retinoblastoma. Two months after completion of systemic chemotherapy, patient presented with tumour recurrence and subretinal fluid and was given the option of enucleation or EBRT. Patient was however lost to follow-up. Other patient was treated with four cycles of intra-arterial chemotherapy and two sessions of intravitreal chemotherapy. She had persistent vitreous seeds. Since parents were not willing for frequent follow-ups, they preferred enucleation. No other case of tumour recurrence was noted in our series.

Mean basal tumour diameter at presentation was $10.9 \mathrm{~mm}$, and at final follow-up was $10.4 \mathrm{~mm}$. Mean tumour thickness at presentation was $7.7 \mathrm{~mm}$, and at final follow-up was $6.5 \mathrm{~mm}$. There was $4.2 \%$ reduction in basal diameter and $14.65 \%$ decrease in tumour thickness at final follow-up. On statistical analysis, there was no significant difference in the baseline and follow-up tumour diameter $(p=0.65)$ and thickness $(p=0.37)$. No patient in our series had metastasis or death.

\section{Discussion}

Cavitary retinoblastoma is a low-grade variant of retinoblastoma characterized by the cystoid or cavitary, translucent spaces within the tumour mass, seen as echolucent lesion on ultrasound and hypofluorescent lesion on FFA [3]. Initially believed to represent tumour liquefaction [4, 5], they are now believed to represent areas of well differentiated photoreceptors [6, 7]. Although, previously they were called as cystic retinoblastoma $[4-6,8]$, due to lack of definite cells lining the cavity, the term 'cavitary retinoblastoma' is now preferred. This article describes the clinical features and treatment outcomes of cavitary retinoblastomas in Indian subjects.

The incidence of cavitary retinoblastoma in literature has been reported to range from 2.3 to $6.8 \%$ [7, 9]. In our study, male;female ratio was $4: 1$. This is in accordance with previous studies where male subjects outnumbered females [1]. A majority of patients had bilateral retinoblastomas, but none of the patients had bilateral cavitary retinoblastoma. However, Rojanaporn et al. [9] have described one case of bilateral cavitary retinoblastoma in their series.

Two patients in our study had retinocytoma, of which, one was diagnosed on the basis of clinical and FFA features and showed positive internal vascularity and staining of scar at the macula, and was observed. The tumour remained stable over a follow-up period of 35 months. The other tumour was initially treated with TTT and in view of inadequate response, was given a trial of CRD for two cycles. But in view of its stable course was redesignated as retinocytoma and observed at regular intervals. It remained stable over a follow-up period of 20 months. Retinocytomas are well differentiated tumours and present as benign, elevated, grey, translucent retinal mass with cottage cheese calcification [7]. They share features similar to those of cavitary retinoblastoma and progress to malignancy in only $10 \%$ of cases [10].

A majority of tumours harboured primary cavities. Secondary cavities were revealed after mean 2 months of systemic or intra-arterial chemotherapy. This is in accordance with the existing literature [7]. With systemic chemotherapy and progressive consolidation of the undifferentiated part of tumour, there is unmasking of the cysts within the tumour mass. It has been previously noted that $60 \%$ of primary tumours involved the fovea [7]. In our study, we could not find any such association. A majority of cavities in our series were located in the tumour periphery. 


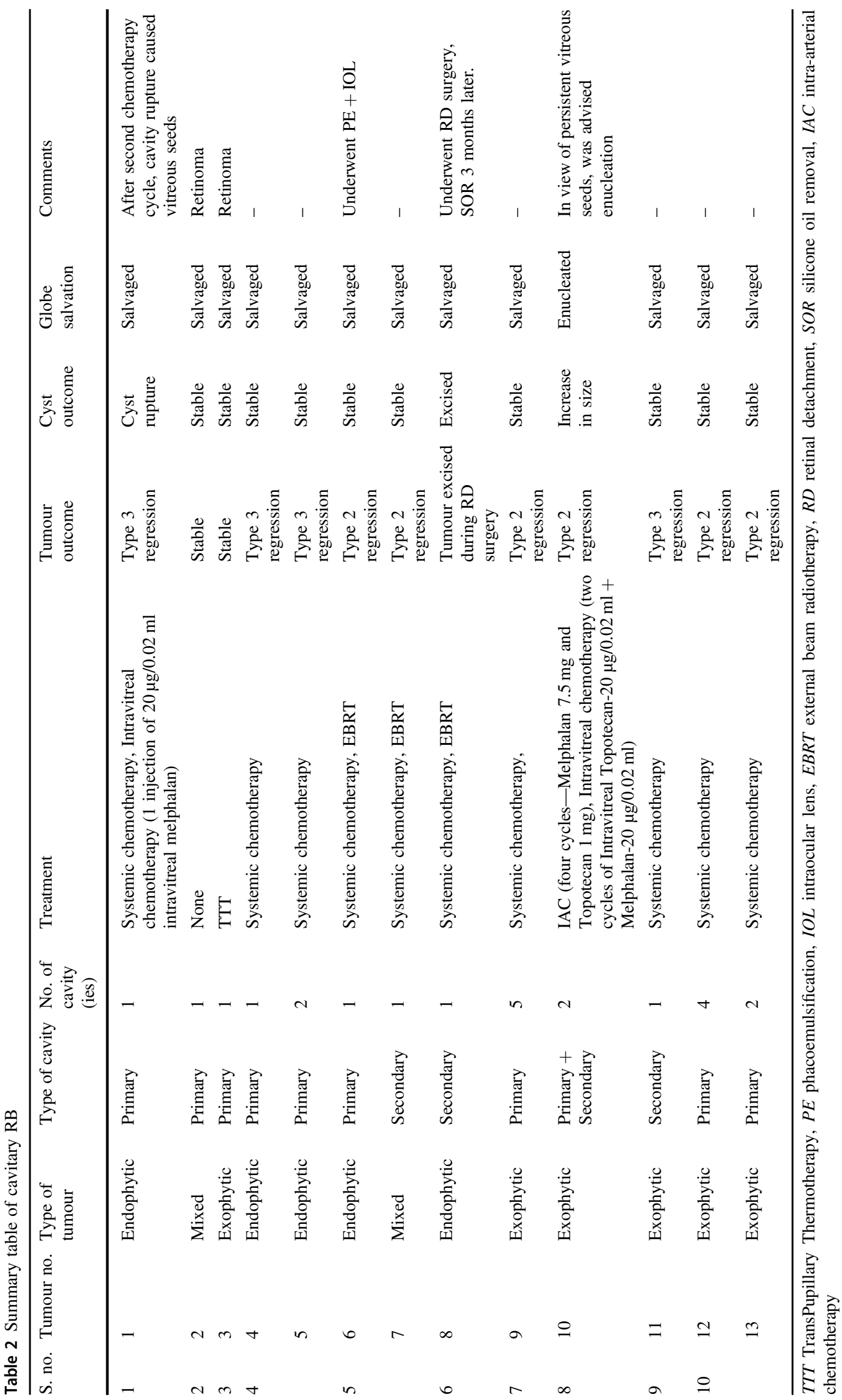




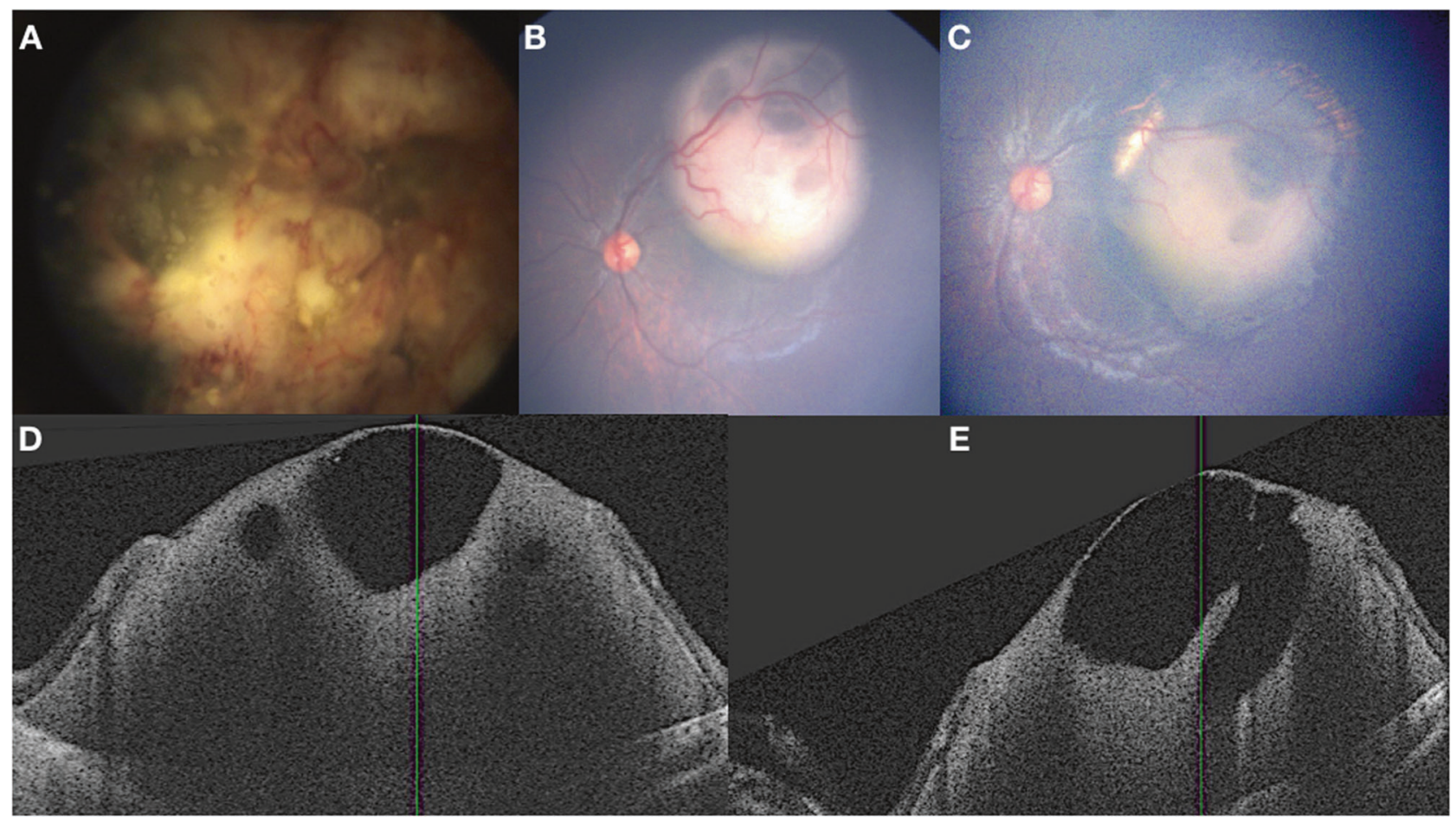

Fig. 2 At presentation, 1-year-old child presented with right eye group E retinoblastoma that underwent enucleation (a). Left eye had group B cavitary retinoblastoma (b) and had focal consolidation with transpupillary thermotherapy (c). Optical coherence tomography showed communicating cavities with intervening septa in inner retina $(\mathbf{d}, \mathbf{e})$

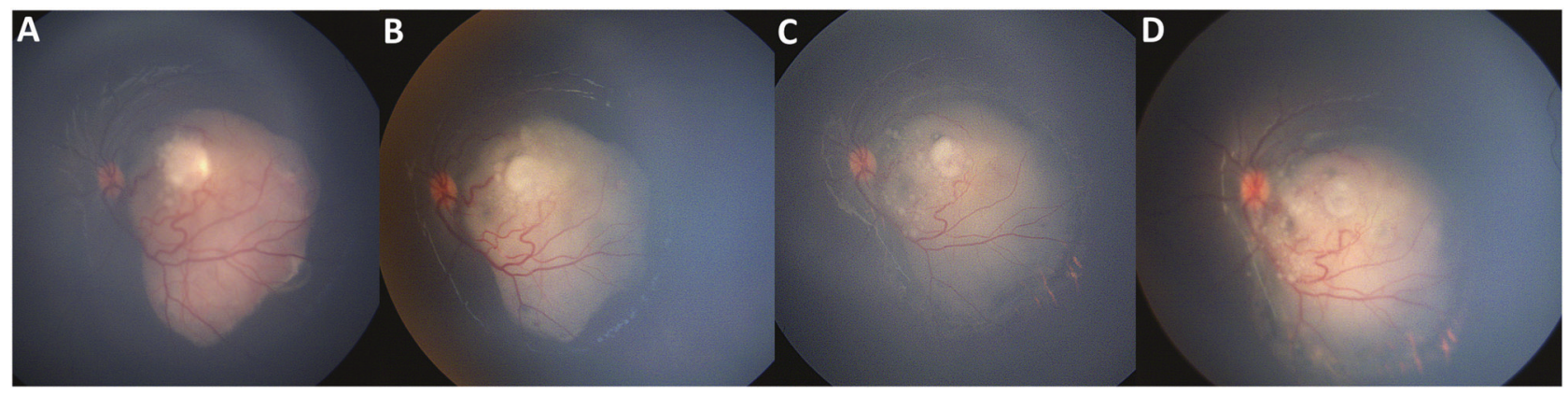

Fig. 3 At presentation (a), fundus photo shows group C RB with predominantly exophytic cavitary tumour with 'sphere' seeds. VS persist after second (b), and third (c) cycle of IAC. Intravitreal melphalan and topotecan were given after third cycle of IAC, with persistence of VS (d)

Interestingly, this is contradictory to the observation made by Mashayekhi et al. [1] who reported that majority of cavities involved central tumour mass.

It has been reported previously [1] that there was absence of vitreous seeds in eyes with cavitary retinoblastoma. Rojanaporn et al. [9] have noted that cavitary tumours are less likely to display intrinsic calcification, subretinal fluid, vitreous seeds, or subretinal seeds. However, our observations contradict this. In our study, four eyes (40\%) had vitreous seeds at the time of presentation. Of these, three eyes did not have other non-cavitary tumours. Chaudhry et al. [7] have noted vitreous seeds in 5\% (one eye with solitary cavitary tumour) and Rojanaporn et al. [9] in $27 \%$ of their cases. The occurrence of other associated features is similar to that noted in previous reports [7,9]. At final follow-up, all patients had resolved or calcified vitreous and subretinal seeds and only one patient had subretinal fluid with tumour recurrence.

Calcification in retinoblastoma is believed to represent the undifferentiated tumour [1, 11-13]. Mashayekhi et al. [1] found calcification to range from 0 to $30 \%$ of cavitary retinoblastomas in their series. In our case series, we found calcification in 6 of $13(46 \%)$ tumours and quantitatively it ranged from 5 to $30 \%$. None of the tumours in our study 
showed type 1 (i.e. total rock salt calcification) or type 4 (i.e. flattened scar formation) regression pattern. It has been documented previously that these tumours do not flatten to scar [7]. Chaudhry et al. [7], however, have noted one tumour with type 1 regression in their series. Majority of tumours showed fish flesh (type 2) regression pattern and few showed mixed (type 3 ) regression pattern, with minimal calcification. This is consistent with the observation of other workers [7]. There was no significant difference in the tumour dimensions at baseline and final follow-up. This is in accordance with the existing literature that cavities impart relative stability to the retinoblastoma tumours and the tumours fail to regress completely after chemotherapy and radiation therapy [1, 7, 14]. Mashayekhi et al. [1] have reported $19 \%$ decrease in basal diameter and $18 \%$ decrease in tumour thickness during a follow-up of 32 months. Rojanaporn et al. [9] noted $22 \%$ reduction in tumour diameter and $29 \%$ reduction in tumour thickness during a follow-up duration of 49 months. Likewise, in our series there was $4.2 \%$ reduction in basal diameter and $14.6 \%$ decrease in tumour thickness at final follow-up. Hence, these tumours tend to retain stable dimensions with little evidence of calcification.

In majority of tumours, systemic chemotherapy along with focal consolidation was used as primary mode of treatment [7, 15-17]. One eye was primarily treated with intra-arterial chemotherapy. The tumour responded well. The role of intra-arterial chemotherapy for cavitary retinoblastomas has been reported before [7]. Intravitreal chemotherapy was needed in two patients during the course of treatment. Lack of response to treatment in cavitary retinoblastomas has been previously documented owing to the well differentiated nature of these tumours [1, 7, 18, 19], and at the same time less rates of recurrence and stable tumour dimensions are also reported. The same is reflected in our study. For one eye, due to apparently inadequate response, the planned systemic chemotherapy was extended. Same patient presented with recurrence and was given the option of EBRT versus enucleation. EBRT due to apparently inadequate response was required in two eyes, while TTT was needed in three tumours. Chaudhry et al. [7] have reported consolidation therapy with TTT in $17 \%$ tumours that were regressed at mean follow-up of 6 months. Mashayekhi et al. [1] have reported a case of nonresponding tumour in their series for which they resorted to EBRT and the tumour was stable over 6 years of follow-up. Thus, uncertainty of the adequate response in cavitary tumours often leads to delivery of aggressive therapy to these tumours. It has also been reported [7] that due to failure of the cavitary retinoblastoma to flatten into a scar, these eyes may land up with multiple examinations and delivered 'over-treatment', causing detrimental effects on vision when tumour is close to fovea. There are reports where after enucleation of the nonresponding tumour to chemotherapy, histopathology revealed areas of well differentiated photoreceptors with no evidence of activity within or at the margins of cavities [1, 7, 9, 12, 13]. In our series, one patient treated with systemic chemotherapy and EBRT, had regressed retinoblastoma, presented 6 months after last treatment session with rhegmatogenous retinal detachment and was managed with vitreous surgery and silicone oil injection; followed by silicone oil removal 3 months later. He had stable fundus with a vision of 6/18 at 13 years of follow-up with no evidence of extraocular or systemic metastasis. There are reports of rhegmatogenous retinal detachment in eyes with inactive retinoblastoma being successfully managed with vitrectomy approach [20, 21].

Cavities were stable in majority of cases. A 2-year male child with group $\mathrm{C}$ cavitary retinoblastoma presented with vitreous seeds, subretinal fluid, and subretinal seeds. After second cycle of systemic chemotherapy, the cavity ruptured spontaneously spewing 'seeds' in the vitreous (Fig. 1). To the best of our knowledge, no such cases of cavity rupture giving rise to vitreous seeds have been reported previously in the literature. Interestingly, none of the tumours in our series showed cavity collapse as is reported in other studies $[1,7]$.

Tumour recurrence was noted in one eye in our series. Rojanaporn et al. [9] have reported tumour recurrence in one (4\%) eye and Chaudhry et al. [7] have reported enucleation in two eyes $(11 \%)$ due to recurrence of vitreous seeds and noncavitary tumour. Mashayekhi et al. [1] have reported that none of their tumours recurred over a period of 32 months.

In summary, while we acknowledge this study is limited by a small sample size, but with adequate duration of follow-up, provides an insight into the long-term behaviour of these rare tumours. Our study reflects the view that tumours with cavities within them tend to be resistant to therapy and maintain relatively stable dimensions. They still need regular follow-up to look for recurrence(s). Our study, however, contradicts the previous observations that these tumours are less likely to display associated vitreous seeds and the cavities tend to be more centrally located in the tumor. We recommend that more work needs to be done to better understand this rare entity.

\section{Conclusion}

Cavitary spaces provide good prognostic value to the retinoblastoma tumour with less chances of recurrence. However, they tend to maintain stable dimensions and fail to regress completely after chemotherapy and radiation therapy. Hence, one must be aware of this entity to avoid delivering cumulatively higher laser energy to the eye and to possibly avoid enucleation in these eyes. However, 
regular follow-up is required to look for tumor recurrence, vitreous seeds, or subretinal fluid.

\section{Summary}

\section{What is known}

- Cavitary retinoblastoma tend to maintain stable tumour dimensions and are less likely to be associated with vitreous seeds.

- Most cavities are centrally located within the tumour.

- Retinoma and retinoblastoma can co-exist in a single tumour.

\section{What this study adds}

- In our series, most tumor cavities were peripheral in location.

- Cavitary tumours have variable presentations, are often associated with vitreous seeds, and in some cases the latter emanates from them as well.

\section{Compliance with ethical standards}

Conflict of interest The authors declare that they have no conflict of interest.

Publisher's note Springer Nature remains neutral with regard to jurisdictional claims in published maps and institutional affiliations.

\section{References}

1. Mashayekhi A, Shields CL, Eagle RC, Shields JA. Cavitary changes in retinoblastoma: relationship to chemoresistance. Ophthalmology. 2005;112:1145-50.

2. Shields CL, Mashayekhi A, Demirci H, Meadows AT, Shields JA. Practical approach to management of retinoblastoma. Arch Ophthalmol. 2004;122:729-35.

3. Palamar M, Pirondini C, Shields CL, Shields JA. Cavitary retinoblastoma: ultrasonographic and fluorescein angiographic findings in 3 cases. Arch Ophthalmol. 2008;126:1598-1600.

4. Samuels B, Fuchs A. Clinical pathology of the eye: a practical treatise of histopathology. New York: Hoeber; 1952. p. 43-4.
5. Ginsberg J, Spaulding AG, Asbury T. Cystic retinoblastoma. Am J Ophthalmol. 1975;80:930-4.

6. Singh AD, Shields CL, Shields JA. Lack of response to chemoreduction in presumed well differentiated retinoblastoma. J Pediatr Ophthalmol Strabismus. 2002;39:107-9.

7. Chaudhry S, Onadim Z, Sagoo MS, Reddy MA. Recognit cavitary retinoblastoma tumors. Implic Manag Genet Anal: Retin. 2018; 38:782-7.

8. Tso MO, Zimmerman LE, Fine BS. The nature of retinoblastoma. I. Photoreceptor differentiation: a clinical and histopathologic study. Am J Ophthalmol. 1970;69:339-49.

9. Rojanaporn D, Kaliki S, Bianciotto C, Iturralde JC, Say EA, Shields CL. Intravenous chemoreduction or intra-arterial chemotherapy for cavitary retinoblastoma: long-term results. Arch Ophthalmol. 2012;130:585-90.

10. Balmer A, Munier F, Gailloud C. Retinoma: case studies. Ophthalmic Paediatr Genet. 1991;12:131-7.

11. Ellsworth RM. In discussion of: Ts'o MO, Zimmerman LE, Fine BS. A cause of radioresistance in retinoblastoma: photoreceptor differentiation. Trans Am Acad Ophthalmol Otolaryngol. 1970; 74:969.

12. Ts'o MO, Zimmerman LE, Fine BS, Ellsworth RM. A cause of radioresistance in retinoblastoma: photoreceptor differentiation. Trans Am Acad Ophthalmol Otolaryngol. 1970;74:959-68.

13. Demirci H, Eagle RC Jr, Shields CL, Shields JA. Histopathologic findings in eyes with retinoblastoma treated only with chemoreduction. Arch Ophthalmol. 2003;121:1125-31.

14. Fuchs SB. A clinical pathology of the eye: a practical treatise of histopathology. New York, NY: Hoeber; 1952. p. 43-44.

15. Shields CL, Meadows AT, Leahey AM, Shields JA. Continuing challenges in the management of retinoblastoma with chemotherapy. Retina. 2004;24:849-62.

16. Shields JA, Shields CL. Management of retinoblastoma. In: Shields JA, Shields CL, eds. Intraocular tumors: an atlas and handbook. Philadelphia, PA: Lippincott Williams \& Wilkins; 2008. p. 334-51.

17. Epstein JA, Shields CL, Shields JA. Trends in the management of retinoblastoma: evaluation of 196 consecutive eyes during 1974-2001. J Pediatr Ophthalmol Strabismus. 2003;40: 196-203.

18. Shields CL, De Potter P, Himelstein BP, Shields JA, Meadows AT, Maris JM. Chemoreduction in the initial management of intraocular retinoblastoma. Arch Ophthalmol. 1996;114: 1330-8.

19. Singh AD, Shields CL, Shields JA. Lack of response to chemoreduction in presumed well differentiated retinoblastoma. J Pediatr Strabismus. 2002;39:107-9.

20. Baumal CR, Shields CL, Shields JA, Tasman WS. Surgical repair of rhegmatogenous retinal detachment after treatment for retinoblastoma. Ophthalmology. 1998;105:2134-9.

21. Madreperla SA, Hungerford JL, Cooling RJ, Sullivan P, Gregor Z. Repair of late retinal detachment after successful treatment of retinoblastoma. Retina. 2000;20:28-32. 